\title{
Leflunomide: less toxic than standard therapy for lupus nephritis?
}

Lupus nephritis (LN) is one of the most serious clinical manifestations of systemic lupus erythematosus; if left untreated, severely affected patients are at high risk of developing end-stage renal disease. The mainstay of treatment for severe $\mathrm{LN}$ is a combination of corticosteroids and immunosuppressive agents such as azathioprine or mycophenolate mofetil. Although this aggressive approach is associated with a survival benefit, adverse effects, including liver damage, gastrointestinal symptoms and leukopenia, are not uncommon, and often lead to the discontinuation of therapy. The results of a study by Zhang et al. suggest that leflunomide, a nonbiologic DMARD used to treat moderate-to-severe rheumatoid arthritis, could be a less-toxic alternative to standard treatment for LN.

Following an initial kidney biopsy, 31 patients with moderate-to-severe LN were administered leflunomide $0.4-0.8 \mathrm{mg} / \mathrm{kg}$ daily together with prednisone $10 \mathrm{mg}$ daily. After 12 months, the investigators saw marked improvements in all clinical and laboratory measures of disease activity, with $58 \%$ of patients achieving partial remission and $42 \%$ achieving complete remission. A second biopsy at 12 months showed significant improvements in active lesions, including glomerular hypercellularity, leukocyte infiltration and immune complex deposits. Furthermore, in $49 \%$ of patients, LN regressed according to WHO criteria. In three patients, the disease stage was reclassified from class V (membranous LN) to class III (focal proliferative LN), and in another three it changed from class III to class II (mesangial proliferative LN). Overall, leflunomide was well tolerated, and only a few patients experienced mild, transient adverse effects.

The results of this small study suggest that leflunomide combined with

\author{
44 ...leflunomide was well \\ tolerated, and only a few patients \\ experienced mild, transient \\ adverse effects 77
}

corticosteroids could be an effective and less-toxic alternative to current therapies for LN, particularly for patients who have relapsed or failed to respond to initial therapy. Further studies in large groups of patients would help to clarify the potential role of this agent in the management of LN.

Negin Nassabeh

Original article Zhang, F. S. et al. The efficacy and
safety of leflunomide therapy in lupus nephritis by
repeat kidney biopsy. Rheumatol. Int. [doi:10.1007/
s00296-0109-0861-3] (2009).

